\title{
Posterior Reversible Encephalopathy Syndrome: An Uncommon Presentation of Celiac Disease
}

\author{
Harish Kumar*, Veer Bahadur Singh, Babu Lal Meena, Subhash Gaur, Sharif Haji Mohammed M., Rahul Singla \\ Department of Medicine, Sardar Patel Medical College, Bikaner (RAJ) India \\ *Corresponding author: drharishsgnr@gmail.com
}

Received August 14, 2015; Revised September 24, 2015; Accepted September 25, 2015

\begin{abstract}
Celiac disease is an autoimmune induced inflammatory disease of small intestine in genetically susceptible individuals by ingestion of gluten. Clinical manifestation of celiac disease is mostly gastrointestinal or atypical non-gastrointestinal symptoms or silent with no symptoms, despite the presence of a characteristic intestinal lesion. Wider spectrum of atypical symptoms include neurological syndrome like migraine, encephalopathy, mylopathy, neuropathy with positive antiganglioside antibodies and symptoms of osteomalacia. Autoimmune disorders occur ten times more frequently in adult patients with celiac disease than in the general population such as thyroid disease, type1 diabetes and Addison's disease etc. If celiac disease remains unrecognized, it can increase the risk of life-threatening complications that are difficult to manage. Herein we are presenting a case of posterior reversible encephalopathy, presenting in department of medicine with seizures. Patient was a diagnosed case of celiac disease.
\end{abstract}

Keywords: celiac disease, gluten, immune system, posterior reversible encephalopathy, seizure

Cite This Article: Harish Kumar, Veer Bahadur Singh, Babu Lal Meena, Subhash Gaur, Sharif Haji Mohammed M., and Rahul Singla, "Posterior Reversible Encephalopathy Syndrome: An Uncommon Presentation of Celiac Disease.” International Journal of Celiac Disease, vol. 3, no. 4 (2015): 159-161. doi: 10.12691/ijcd-3-4-3.

\section{Introduction}

Celiac Disease (CD) is common throughout the world and affects between approximately one in 100 and one in 300 individuals. It is characterizes by small intestinal mucosal injury following the dietary ingestion of gluten. Proteins in the dietary cereals grains, wheat, rye, barely are the major environmental factor required for disease activation. [1] The disease occurs in about $1 \%$ of general population. It may be diagnosed in any age and can affect any system. [2] CD goes is associated with the prevalence of HLA-DQ2, and also to a minor degree with that of DQ8. CD presented with typical presentation of weight loss and diarrhea but we know now that CD occurs frequently without gastrointestinal symptoms. Atypical presentation of $\mathrm{CD}$ is neurologic and psychiatric disorders including, epilepsy, dementia, and depression, mylopathy, myopathy etc. Posterior reversible encephalopathy syndrome (PRES) is a clinic-radiological diagnosis described by Hinchey et al. [3] PRES is characterized by seizure activity, alter sensorial, headache, vomiting and impairments of vision. PRES is caused by a vast array of condition. The underlying condition which activate the immune response, inflammatory response, endothelial cell and vascular dysfunction, predispose to PRES such as sepsis, after exposure to immunosuppressants, in autoimmune/ renal/ hypertensive diseases. [4,5,6,7] Herein we are report a case of PRES associated with CD presented with seizure disorder.

\section{Case Report}

A 17 year old male was admitted in department of medicine with the chief complain of acute onset of seizures and loss of consciousness. Patient had the history of two to three episode of involuntary movement of upper and lower limbs which started from right upper limbs and becomes generalized. Seizures associated with loss of consciousness, micturition, and tongue bite. Antiepileptic treatment was started as soon as possible. After stabilized the patient, detail history was taken. As history was given by their mother, patient was discharged two days back from department of medicine with the diagnosis of $\mathrm{CD}$ in stable condition. No past history of any neurological disorder was present. No history of fever, headache, vomiting, syncope, blurring of vision was present.

On examination patient was unconscious with Glasgow Coma Scale $8 / 15$, pupil mid dilated normally reactive to light and planter bilateral extensor. Conservative management with antiepileptic drugs was started. Serum samples for hematological and biochemical investigation was sent. Patient was shifted for magnetic resonance imaging (MRI) brain.

On admission blood pressure was 140/90 $\mathrm{mmHg}$ by right brachial artery, pulse rate 110/minute with ECG showing sinus tachycardia, respiratory rate after stabilization 18/minute regular abdomino-thoracic, saturation $98 \%$ on room air. Both lung fields were clear on auscultation. Cardiac auscultation was also normal. 
The results of blood analysis were as follows: $\mathrm{Hb} 7 \mathrm{~g} / \mathrm{dl}$, WBC 3200, platelets 1.86 , total bilirubin $3.2 \mu \mathrm{mol} / 1$, total proteins 4.5 g/l, albumins 2.3 g/l, ALT 74 U/l, AST 138 $\mathrm{U} / \mathrm{l}$. Renal function was in normal limit. Electrolytes were normal. Other laboratory investigations including erythrocyte sedimentation rate (ESR), C-reactive protein (CRP), thyroid function tests, and creatinine kinase were normal. Antinuclear antibodies (ANA) and anti-DNA were negative. Immunological markers for hepatitis $B$ and
C, HIV, CMV, HSV and dengue were negative. Cerebrospinal fluid was also normal in investigation.

As the patient had the old history of mild diarrhea, weight loss and anemia patient was investigated for malabsorption. Antigliadin IgA and IgG antibodies [IgA:28.5 (normal values 0-12), IgG: 96.2 (normal values 0-12) ] were found to be elevated and biopsy confirmed a diagnosis of CD. Biopsy showing flattening of mucosa with lymphoplasmocytic infiltration of lamina propria (Figure 1).

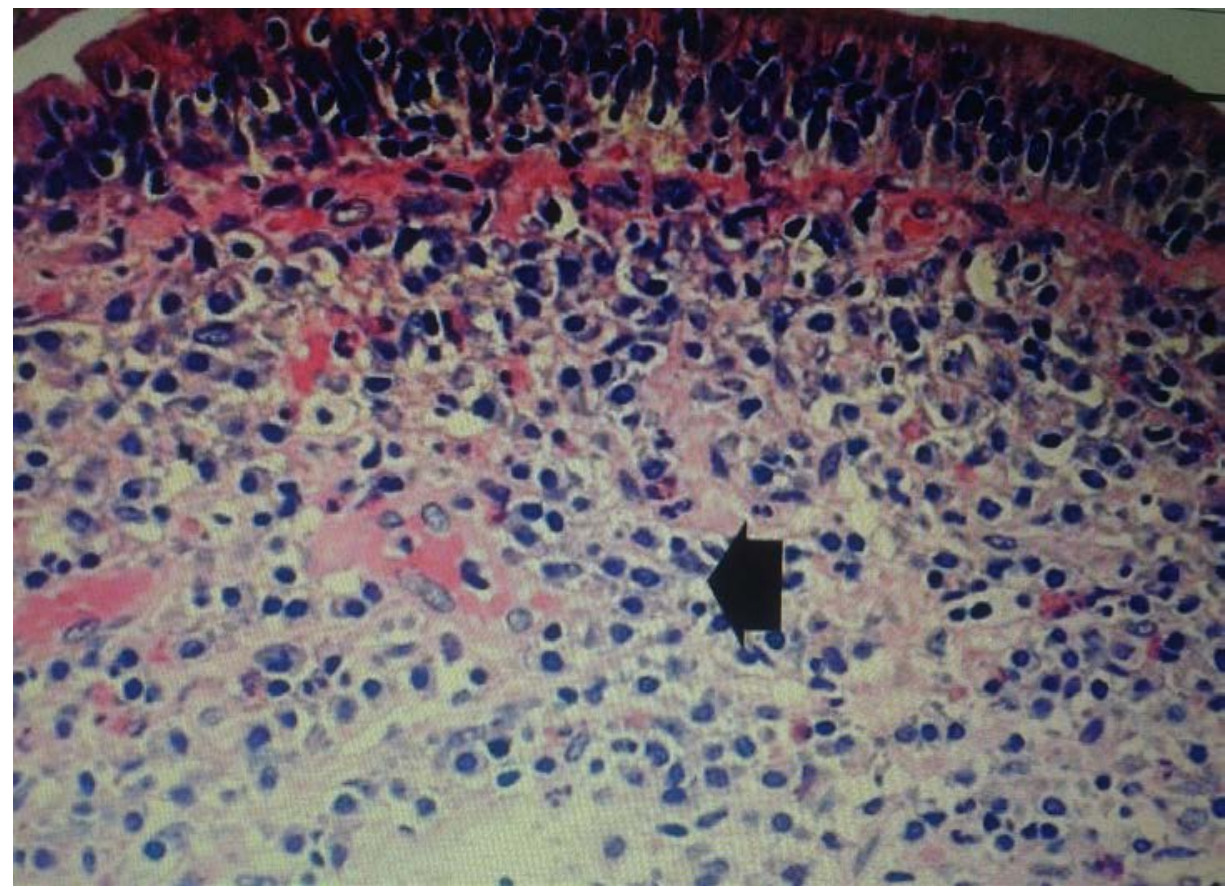

Figure 1. The long arrow at the top shows intraepithelial lymphocytes with loss of brush border. The broad arrow shows small lymphocytes, plasma cells, and eosinophils, flattened surface epithelium at the top

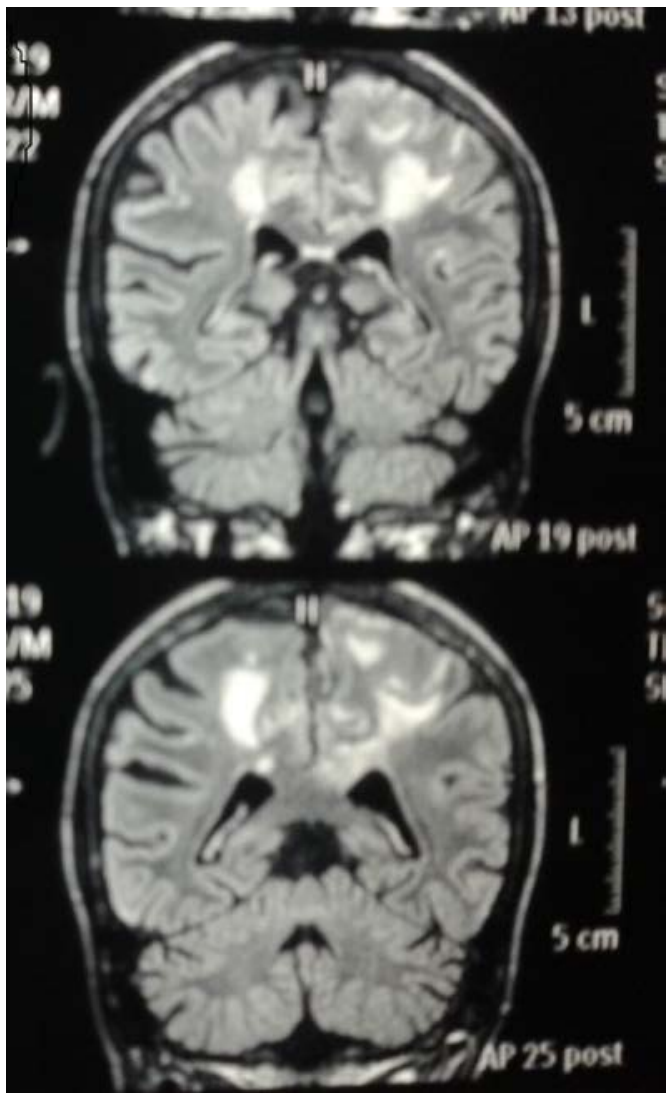

Figure 2. MRI brain
MRI brain revealed multiple altered signal intensity areas in bilateral superior parietal and bilateral frontal region. There is diffuse fluid attenuated iversion recovery hyperintensity incortical and subcortical region. (Figure 2) The patient was treated with antiepileptic, manitol, antibiotics and other supportive measures, with continuously monitored: blood pressure, pulse rate, oxygen saturation, temperature. Patient was treated for 7 days, sensorial was improved and seizure was subside and discharged with a gluten-free diet and also given vitamin $\mathrm{D}$, calcium, and iron supplementation.

\section{Discussion}

PRES is the neuroradiological entity with the sign and symptoms of impairments of mental status, headache, visual impairment, vomiting and seizures with characteristic MRI scan finding. PRES is associated with predisposed clinical condition like preeclampsia, toxemia in pregnancy, chronic kidney disease, chronic glomerulonephritis, hemolytic uremic syndrome and immunomodulating agent which leads to immune system activation. The condition which most commonly associated with PRES are toxic agent, infection/sepsis/septic shock, hypertension, eclampsia/preeclampsia, autoimmune disease and other rare condition like hypercalcemia, Guillain-Barre syndrome, tumor lysis syndrome, sickle cell disease, Porphyria and Cushing syndrome. The intensity and severity vary may require intensive care unit admission. The combinations of 
clinical manifestation and radiological criteria established the diagnosis. The path physiology of PRES is not fully understood. One involves impaired cerebral auto regulation responsible for increase in cerebral blood flow whereas other involves endothelial dysfunction with cerebral hypo-perfusion. In both condition blood brain barrier dysfunction with vasogenic edema is the main cause of cerebral blood perfusion abnormality. [6] It is supposed to rapidly rise of blood pressure leads to (which already in state of compromised blood brain barrier) forced dilatation of blood vessels ,cerebral hyperperfusion, impaire cerebral autoregulation and vasogenic cerebral edema in hypertensive patient. [6,7,8,9] It can develop without significant rise in blood pressure, may be due to impaired autoregulation capacity or severe endothelial injury.

CD is commonly presented with abdominal pain, diarrhoea, mailase, and weight loss. Atypical presentation of $\mathrm{CD}$ is uncommon however some cases has been reported with atypical presentation like osteomalacia, neuropathy, and mypathy.[10] In hand case is presented with generalized tonic clonic seizure which further diagnosed as PRES.

Our patient is a known case of CD is thought to result from the activation of both a cell-mediated (T-cell) and humoral (B-cell) immune response on exposure to the glutens (prolamines and glutenins) of wheat, barley, rye, and (rarely) oats, in a genetically susceptible person and an association with certain type II human leukocyte antigens (HLA). HLA-DQ2 is found in up to 95\% of CD patients, while most of the remaining patients have HLADQ8.

The presence of autoantibodies to the connective-tissue element surrounding smooth muscle known as endomysium is highly specific for CD. The target of these autoantibodies is now known to be the enzyme tissue transglutaminase (tTG). This enzyme may play a prominent role in the pathogenesis of CD by deamidating gliadin, resulting in a greater proliferative response of gliadin-specific T-cells, which contributes to mucosal inflammation and further B-cell activation in patients with HLA-DQ2 or -DQ8.

The path physiology of PRES in a patient of CD is thought to be related to immune system activation. The immune system activation ( $T$ cells) leads to release of various mediator like histamine, free redicals, bradykynin, NO, and arachidonic acid by endothelial cells. [11] Proinflamatory cytokines like tumor necrosis factor (TNF), IL-6, IL-1 and interferon increased by these mediators. Leucocyte trafficking increases via the release of adhesion molecules. [11] These all complex mechanism leads to upregulation of endothelial surface antigen and the release of endothelin affect the local vascular tone. [12] All these changes result of vasoconstriction and hypoperfusion. Studies [7,13] support this hypothesis. Immunohypothesis is the result of PRES in a case of CD, which explain the cause of PRES in CD.

\section{Conclusion}

Early diagnosis and treatment of CD is important. Atypical presentations are not rare but PRES is a rarer manifestation of CD.

\section{References}

[1] Kangnoff MF, Celiac disease. A gastrointestinal disease with environmental, genetic, and immunologic components. Gastroentero lClin North Am. 1992; 21: 405-425.

[2] Green PH, Cellier C. Celiac disease: N Engl J Med. 2007; 357: 1731-1743.

[3] Hinchey J, Chaves C, Appignani B, Breen J, Pao L, Wang A, et al. A reversible posterior leukoencephalopathy syndrome. N Engl J Med 1996; 334: 494-500.

[4] Bartynsky WS. Posterior reversibile encephalopathy syndrome, part 1: fundamental imaging and clinical features. AJNR Am J Neuroradiol 2008; 29:1036-1042.

[5] Bartinsky WS, Bordman JF, Ziegel RR, Shadduck RK, Lister J. Posterior reversibile encephalopathy syndrome in infection, sepsis and schock. AJNR Am J Neuroradiol 2006; 27: 2179-2190.

[6] Bartynsky WS. Posterior reversible encephalopathy syndrome, part 2: controversies surrounding pathophysiology of vasogenic edema. AJNR Am J Neuroradiol 2008; 29: 1043-1049.

[7] Brubaker LM, Smith JK, Lee YZ, Lin W, Castillo M. Hemodynamic and permeability changes in posterior reversible encephalopathy syndrome measured by dynamic susceptibility perfusion-weighted MR imaging. AJNR Am J Neuroradiol 2005; 26: 825-830.

[8] Wagner SJ, Acquah LA, Lindell PE. Posterior reversibile encephalopathy syndrome and eclampsia: pressing the case for more aggressive blood pressure control. Mayo ClinProc 2011; 86: 851-856.

[9] Fugate JE, Claassen DO, Cloft HJ, Kallmes DF, Kozak OS, Rabinstein AA et al. Posterior reversible encephalopathy syndrome: associated clinical and radiologic findings. Mayo ClinProc 2010; 85:427-432.

[10] Byrne MF, Razak AR, Leader MB, Sheehan KM, Patchett SE. Disabling osteomalacic myopathy as the only presenting feature of coeliac disease. Eur J Gastroenterol Hepatol.2002; 14: 1271-1274.

[11] Stanimirovic D, Satoh K. Inflammatory mediators of cerebral endothelium: a role in ischemic brain inflammation. Brain Pathol. 2000; 10: 113-126.

[12] Narushima I, Kita T, Kubo K. Highly enhanced permeability of blood-brainbarrier induced by repeated administration of endothelin-1 in dogs and rats. PharmacolToxicol. 2003; 92: 21-26.

[13] Bartynski WS, Boardman JF. Catheter angiography, MR angiography, and MR perfusion in posterior reversible encephalopathy syndrome. AJNR Am J Neuroradiol. 2008; 29: 447-455. 